\title{
Motivating the Students' Creativity for Learning through Sharing Experience with Teacher in Laboratories
}

\author{
DengGuang Yu, Deng Pan, Xian Ying Wang and Feng Tian \\ School of Materials Science \& Engineering, University of Shanghai for Science and Technology, Shanghai 200093, China \\ ydg017@usst.edu.cn; feiyu.dpan@gmail.com
}

\begin{abstract}
The present study investigates the motivating effect on the students' creativity for learning. Three scientific methods (ultraviolet-visible spectrophotometry, scanning electron microscope and transmission electron microscopy) with each given three lessons are purposefully arranged in the laboratory and classroom differently. The results for the final examination demonstrated that the experimental lessons in the laboratory can effectively motivate the students' creativity for learning. The advantages provided by the laboratory include a free and creative environment, the personal attention that impresses them most, more stimuli for motivating the students' thinking, expressing the knowledge in a right way, and most importantly sharing experience with the teachers in laboratory. It is recommended that more experimental lessons can be taken in laboratory in higher education for motivating the students' creativity for learning and also fostering the students' innovation ability.
\end{abstract}

Index Terms - Learning, Higher education, Creativity, Experience, Experiments

\section{Introduction}

To some extent, learning is a creative process that involves students making information relevant by linking prior knowledge and new knowledge in an individually meaningful format [1]. Wilson et al. state that when students are able to relate their personal experiences to the course content, they tend to be more active in the learning process [2]. However and Carter report that when teachers include their personal experiences and allow students to share personal experiences in the classroom, students have a greater opportunity to relate to the course [3].

Most of the reports focus on methods of teaching creativity in the classroom, and some emphasize the importance of building teacher-student relationships in a creative environment [4-6]. Morganett states that quality teacherstudent relationships can encourage students to be active learners in the classroom, which will foster creativity [7]. However, the scientific laboratory should be a better place than the classroom to motivate the students' enthusiasm about new knowledge, particularly with some interesting experiments that have a close relationship with our daily life.

On the other hand, the teachers often have abundant experience about scientific researches. The students would be benefited a lot provided the teachers can share their experience effectively with the students. Here, we investigate how the teachers can motivate the students' creativity to achieve new knowledge through sharing their teachers' experience about scientific experiments.

\section{Design of the Project}

There are three experiments, i.e. ultraviolet-visible spectrophotometry (UV), scanning electron microscope (SEM), and transmission electron microscope (TEM). Each of them was taught with the same time periods of three lessons. Two, one and none of the lessons were given in the laboratory for UV, SEM and TEM, respectively. A schematic diagram about the arrangement and the procedure is shown in Fig. 1. All the contents were taught in the same procedure, i.e. the principle of the method, the instrument and the related operation, and the potential applications (Fig. 1).

\begin{tabular}{|c|c|c|c|c|}
\hline \multirow{4}{*}{ 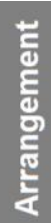 } & Methods & Lesson 1 & Lesson 2 & Lesson 3 \\
\hline & UV & In classroom & In laboratory & In laboratory \\
\hline & SEM & In classroom & In classroom & In laboratory \\
\hline & TEM & In classroom & In classroom & In classroom \\
\hline & \multirow{2}{*}{\multicolumn{2}{|c|}{ Principle }} & \multicolumn{2}{|c|}{$r------)$} \\
\hline & & & n & \\
\hline & \multicolumn{2}{|c|}{ Applications } & Operati & ion \\
\hline
\end{tabular}

Fig. 1 The arrangement of the lessons and the procedure for lesson

In the UV lesson in the classroom, the main knowledge points were simply preached and would be expanded in the laboratory. UV refers to absorption spectroscopy or reflectance spectroscopy in the ultraviolet-visible spectral region. This means it uses light in the visible and adjacent (near-UV and near-infrared) ranges. The absorption or reflectance in the visible range directly affects the perceived color of the chemicals involved [8].

SEM is a type of electron microscope that produces images of a sample by scanning it with a focused beam of electrons. The electrons interact with atoms in the sample, producing various signals that can be detected and that contain information about the sample's surface topography and composition. The electron beam is generally scanned in raster scan pattern, and the beam's position is combined with the detected signal to produce an image. SEM can achieve resolution better than 1 nanometer. Specimens can be observed in high vacuum, in low vacuum, (in environmental SEM) in wet conditions and at a wide range of cryogenic or elevated 
temperatures. The most common mode of detection is by secondary electrons emitted by atoms excited by the electron beam. The number of secondary electrons is a function of the angle between the surface and the beam. By scanning the sample and detecting the secondary electrons, an image displaying the tilt of the surface is created [9].

TEM is a microscopy technique in which a beam of electron is transmitted through an ultra-thin specimen, interacting with the specimen as it passes through. An image is formed from the interaction of the electrons transmitted through the specimen; the image is magnified and focused onto an imaging device, such as a fluorescent screen, on a layer of photographic film, or to be detected by a sensor such as a CCD camera. TEMs are capable of imaging at a significantly higher resolution than light microscopes, owing to the small de Broglie wavelength of electrons. This enables the instrument's user to examine fine detail — even as small as a single column of atoms, which is thousands of times smaller than the smallest resolvable object in a light microscope. TEM forms a major analysis method in a range of scientific fields, in both physical and biological sciences. TEMs find application in cancer research, virology, materials science as well as pollution, nanotechnology, and semiconductor research [10].

\section{Results and Discussion}

In the final examination, three questions were put forward to the 39 students. The details of these questions are listed as follows:

Q1 for UV: Phenol is an aromatic organic compound with the molecular formula $\mathrm{C}_{6} \mathrm{H}_{5} \mathrm{OH}$. Phenol and its vapors are corrosive to the eyes, the skin, and the respiratory tract. It may cause harmful effects on the central nervous system and heart, resulting in a series of diseases. It is a common pollutant in the drinking water, which a content of $2 \mu \mathrm{g} / \mathrm{mL}$ is the upper limitation according to the Chinese Government Regulations. Please design a method to determine if the drinking water in the University of Shanghai for Science and Technology (USST) is qualified based on your knowledge of UV Spectroscopy and give your reasons. If a standard line of phenol in water is $\mathrm{A}=0.034 \mathrm{C}(\mu \mathrm{g} / \mathrm{mL})+0.005$ within the range of $1 \sim 50 \mu \mathrm{g} / \mathrm{mL}$ and the sample of the drinking water from USST has an absorbance of 0.057 , does the drinking water meet the criterion? (Shown in Fig. 2a)

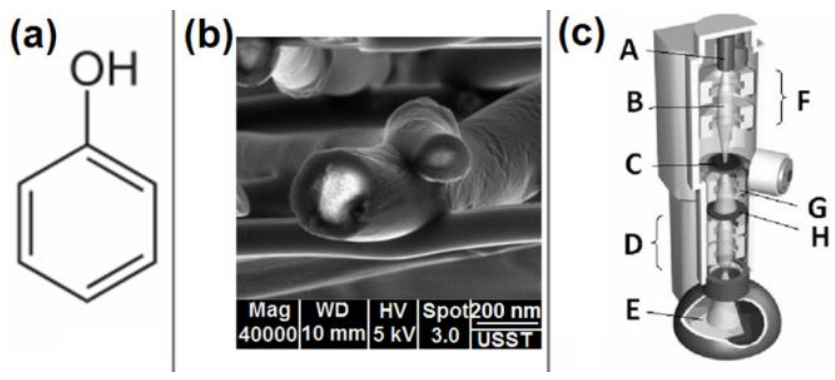

Fig.2 Pictures for examination questions: a) Molecular formula of phenol; b) An SEM image of core-shell nanofibers; c) A schematic diagram of TEM.
Q2 for SEM: Read the images collected from scanning electron microscope (SEM) about core-shell nanofibers. Why this is an image of SEM but not transmission electron microscope (TEM) ? What are the operational conditions? Explain the possible reasons that the core and shell parts have a different gray level. Why the samples of SEM should be electrically conductive? (Shown in Fig. 2b)

Q3 for TEM: Give the name of each part, for example A is Electron source, and what is B to H. Explain the working principle of TEM. How to prepare TEM samples of metal nanoparticles? (Shown in Fig. 2c)

From the standpoint of the test providers, the question of UV (Q1) is the most difficult one because it needs the students to narrate its principle, its operation and also design a way for possible application. The question of TEM (Q3) is the easiest one, in which the students are only required to point out the components of TEM machine, narrates its principle and how to prepare the sample. The question of SEM (Q2) is the easier than UV because the content does not involve systematic applications, but is more difficult than TEM because of reading SEM image and providing explanations to the results. The detailed answers for the questions are given and analysed as follows:

A1 for UV: UV spectroscopy can be used to determine if the quality of drinking water in the University of Shanghai for Science and Technology. The Principle of UV-vis spectroscopy is the Beer-Lambert Law $\mathrm{A}=\log \left(\mathrm{I}_{\mathrm{O}} / \mathrm{I}\right)=\varepsilon c l$, where $A$ is the measured absorbance, $I_{0}$ is the intensity of the incident light at a given wavelength, $I$ is the transmitted intensity, $L$ the path length through the sample, and $c$ the concentration of the absorbing species. For each species and wavelength, $\varepsilon$ is a constant known as the molar absorptivity or extinction coefficient.

The procedures are listed as follows: (1) Selecting maximum absorbance wavelength of phenol -- find the suitable wavelength for the analysis; (2) Building calibration curve: preparing a series phenol solutions with different concentrations, measure their absorbance, and then a plot of either the absorbance or \%transmittance against wavelength can be made for phenol; (3) The absorbance of the phenol in the drinking water of USST is then determined and the prepared calibration plot is used for the determination of its concentration.

According to the standard line, i.e. $\mathrm{A}=0.034 \mathrm{C}(\mu \mathrm{g} / \mathrm{mL})+$ 0.005 , the concentration of phenol in the drinking water in USST is $C=(0.057-0.005) / 0.034=1.53 \mu \mathrm{g} / \mathrm{mL}<2.0 \mu \mathrm{g} / \mathrm{mL}$, thus the drinking water in USST meets the criterion.

A2 for SEM: The SEM produces images of a sample by scanning it with a focused beam of electrons. The electrons interact with atoms in the sample, producing various signals that can be detected and that contain information about the sample's surface topography and composition. Form the images, it is clear that it provide surface topographies of the nanofibers' surface and also their cross-section, such as the wrinkles and the concentric black and white circles. From the image, the operational conditions are listed as follows: 
Accelerating voltages (HV) is $5 \mathrm{kV}$; Working distance (WD) is $10 \mathrm{~mm}$; Spot size is 3.0; and the magnification is 40,000 .

In the SEM process, the electron beam hits the specimen, producing, among others, secondary and backscattered electrons, these electrons are collected by a detector, converted to a voltage, and amplified. The most common mode of detection is by secondary electrons emitted by atoms excited by the electron beam. Under the same conditions, the core and shell parts have different responses to the incident beams due to their different compositions, and thus have varied gray levels. Most probably, the core parts consist of metal and the shell parts consist of organic polymers, and correspondingly the core parts generate more secondary electrons, show a whiter colour.

For conventional imaging in the SEM, specimens must be electrically conductive, at least at the surface, and electrically grounded to prevent the accumulation of electrostatic charge at the surface. Nonconductive specimens tend to charge when scanned by the electron beam, and especially in secondary electron imaging mode, this causes scanning faults and other image artifacts.

A3 for TEM: (1) The name of each part: $A$ is Electron source; B is Electron beam; C is Sample; D is Projector lenses; $\mathrm{E}$ is viewing screen; $\mathrm{F}$ is Condenser lenses; $\mathrm{G}$ is Objective Lens; and $\mathrm{H}$ is Objective Aperture.

(2) A electron beam is focused by 2 condenser lenses, restricted by a condenser aperture; The beam strikes a specimen and part of it is transmitted; This transmitted portion is focused by objective lens into an image; The image is passed down through enlarge lenses and a projector lens, being enlarged all the way; The image strikes the phosphor image screen and light is generated, allowing user to see the image.

(3) i) preparing a suspension of the metal nanoparticles in a solvent such as chloroform or methanol; ii) Pipette a drop of suspension onto a support grid with a Carbon film coating; iii) Solvent will evaporate leaving powder dispersed on the grid; iv) Allow to dry and observe.

Interpretation of the results The statistical results of the three examination questions are shown in Fig. 3. The average scores (with mean deviations) were $80.2 \pm 10.2$ (Fig. 3a), 80.9 \pm 3.7 (Fig. 3b), and $83.8 \pm 6.7$ (Fig. 3c) for TEM, SEM and $\mathrm{UV}$, respectively. Although the question for UV is the most difficult one, the students provide the best results about the understanding and applications of UV. In sharp contrary, the question for TEM is the easiest one; the students provide the poorest results about the understanding of TEM.

In the students' answers about the UV question, almost all the students can correctly write out the principle of UV, i.e. the Beer-Lambert Law; Over $80 \%$ of the students can design the procedures for detecting the content of phenol in the drinking water of USST. And almost all the students can calculate the final results and give their judgments about if the drinking water meets the national standard.

In the students' answers for the SEM question, most students can clearly write out the reason why it is an SEM image, the operational conditions, and explained why the samples of SEM should be electrically conductive. These contents are taught in the classroom and also gone over in the laboratory. However, over half of the students show a poor performance about the explanation of the possible reasons that the core and shell parts have a different gray level.

In the students' answers for the TEM question, most of the students can tell the name of each component from the schematic diagram. But some students explain the working principle of TEM ambiguously and can not narrate the totally right procedures for the preparation of TEM samples of metal nanoparticles.

From the above-mentioned results, it is clear that the more time the students spent on the laboratory, the better result they grasped the related knowledge. The laboratory provides not only an interactive opportunity between the students and the teacher based on the real instruments, but also give a creative environment. In this environment, it is easy for the teachers to encourage creative thinking and encourage students to give their own judgments, emphasized the capability of creativity, and find out more stimuli for creative thinking through experimental practices.

The lessons in the laboratory not only can be used to review the knowledge given in the classroom, but also help the student to express what they know if they can relate the experiments to information from their last lesson during the discussion between the teachers and students, and also among the students themselves.
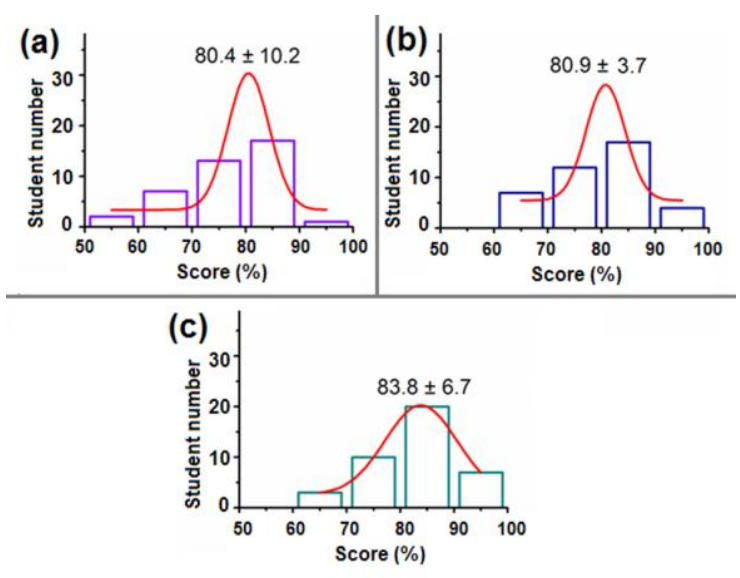

Fig. 3 The test statistics of the three examination questions on (a) TEM; (b) SEM; and (c) UV

\section{Conclusions}

The present study investigates the influence of lesson arrangement on the students' performance in their study. The results clearly demonstrate that the experimental lessons in the laboratory can effectively motivate the students' creativity for learning. The advantages provided by the laboratory include: 1) a free and creative environment; 2) the personal attention that impresses them most; 3 ) more stimuli for motivating the students' thinking; 4) expressing the knowledge in a right way; and also 5) sharing experience with teacher in laboratory. Thus 
it is recommended that more experimental lessons can be taken in laboratory in higher education for motivating the students' creativity for learning and also fostering the students' innovation ability.

\section{Acknowledgment}

This work was supported by the National Science Foundation of China (No. 51373101), the China NSFC/UK Royal Society cost share international exchanges scheme (No.51411130128), the Natural Science Foundation of Shanghai (No. 13ZR1428900) and the Key Project of the Shanghai Municipal Education Commission (No.13ZZ113).

\section{References}

[1] A. J. Starko, "Creativity in the classroom: Schools of curious delight" New York, NY: Longman Publishers, 1995.

[2] R. Wilson, L. Wood, and J. Gaff, "Social-psychological accessibility and facultystudent interaction beyond the classroom," Sociol. Edu., vol. 47, pp.74-92, 1974.
[3] M. Carter, "Training teachers for creative learning experiences," Exchange, 1992, vol.5, pp.38-40, 1992.

[4] Y.J. Dori and J. Belcher, "How does technology-enabled active learning affect undergraduate students' understanding of electromagnetism concepts?" J. Learn. Sci., vol. 14, pp.1-50, 2004

[5] N. Dabbagh and A. Kitsantas, "Personal Learning Environments, social media, and self-regulated learning: A natural formula for connecting formal and informal learning," Intern. High. Edu., vol.15, pp. 3-8, 2012.

[6] T. Valtonen, S. Hacklin, P. Dillon, M. Vesisenaho, J. Kukkonen, and A. Hietanen, "Perspectives on personal learning environments held by vocational students," Comput. Edu., vol. 58, pp.732-739, 2012.

[7] L. Morganett, "Good teacher-student relationships: A key element in classroom motivation and management," Education, vol.112, pp.260264, 1991.

[8] Anonymity,http://en.wikipedia.org/wiki/Ultravioletvisible spectrophotometry, 21-May-2014.

[9] Anonymity, http://en.wikipedia.org/wiki/Scanning_electron_microscope, 21-May-2014.

[10] Anonymity, http://en.wikipedia.org/wiki/Transmission_electron_microscope, 21May-2014. 